\title{
Predictive Factors for Sensory Processing Disorders
}

\author{
Gandara-Gafo $\mathrm{B}^{1,3 *}$, Delgado-Lobete $\mathrm{L}^{3 *}$, Montes- \\ Montes $\mathbf{R}^{3,4}$, Vila-Paz $\mathbf{A}^{3}$ and Santos-del-Riego $\mathbf{S}^{2,3}$ \\ ${ }^{1}$ Department of Health Sciences, University of A Coruna, \\ Spain \\ ${ }^{2}$ Department of Physiotherapy, University of A Coruna, \\ Spain \\ ${ }^{3}$ University of A Coruna, Health Integration and \\ Promotion Research Unit (INTEGRA SAUDE), Spain \\ ${ }^{4}$ University of A Coruna, Centre for Information and \\ Communications Technology Research (CITIC), Spain \\ *Corresponding author: Gandara-Gafo B, \\ Department of Health Sciences, University of A Coruna, \\ Faculty of Health Sciences, 15006, A Coruna, Spain \\ Delgado-Lobete L, Health Integration and Promotion \\ Research Unit (INTEGRA SAÚDE), University of A \\ Coruna, 15011, A Coruna, Spain
}

Received: January 19, 2021; Accepted: February 23, 2021; Published: March 02, 2021

\section{Introduction}

First described by Jean Ayres [1-4], sensory processing problems include various disorders such as sensory reactivity, difficulty in interpreting and using sensory information from the environment to regulate behaviour [5]. Sensory reactivity problems are manifested through atypical behaviours in response to sensory stimuli and affect participation in daily activities, learning, play and social relationships [6]. Prevalence studies have found that, in countries such as the United States, $13.7 \%$ of children attending regular day care centers show alterations in sensory processing [7]. This is similar to data found in Spain with a prevalence of between $14 \%$ and $28 \%$ [8] in children aged 3-10 years. The prevalence of children with sensory reactivity problems increases when specific diagnostic populations such as Autism Spectrum Disorders (ASD) are analyzed with prevalences ranging from $45 \%$ to $96 \%[9,10]$. Within Ayres Sensory Integration (ASI) theory, researchers in the field have studied possible prenatal and perinatal causes that can trigger a sensory processing problem $[5,11]$. From this perspective, the mother's experiences during pregnancy are a factor that seems to be related to possible problems in the baby's sensory processing. Among prenatal complications, the study by Schneider et al. [11] with rhesus monkeys showed that prenatal exposure to alcohol, stress or increased cortisol during pregnancy induce sensory processing problems. Other studies indicate that both neonatal complications and complications during delivery, such as fetal distress, jaundice and prolonged labour, can trigger sensory problems [5]. Prematurity is another variable related to IS problems $[5,12,13]$. May-Bensson et al. [5] found that between 12 and $16 \%$ of children born prematurely show sensory reactivity problems; consequently, prematurity is considered one of the essential variables in the study of perinatal factors in children with SI dysfunction.
Prenatal and perinatal complications can lead to newborn hospitalization. In this connection, sensory deprivation resulting from prolonged stays in neonatal intensive care units puts premature or critically ill infants at increased risk of sensory reactivity problems [14].

The relationship between prenatal and perinatal problems with sensory processing difficulties points to the need to identify such problems at an early age in order to provide interventions that enhance development and participation in daily life. Therefore, this study aims to identify possible differences in sensory processing in children with sensory problems (both with and without prenatal and perinatal problems).

\section{Materials and Methods}

\section{Instruments}

To identify problems in sensory processing, the Sensory Profile-2 (SP-2) [15] parent-reported sensory questionnaire was used for children aged 3-14 years. This instrument analyses the processing of six sensory factors: auditory, visual, tactile, movement, body position and oral, as well as three factors relating to alterations in sensory processing: behavioural, emotional and attentional. The SP-2 follows Dunn's Model [16], which describes the problems deriving from sensory processing, such as low registration, sensory seeking, sensory sensitivity and sensory avoiding. The SP-2 provides adequate metric data and has obtained reference values for children in the United States and Spain [15].

In order to detect prenatal and perinatal problems in children with sensory processing problems, the principal investigator (first author) extracted data relating to the study variables from the clinical interviews, which were recorded in the medical histories drawn up during the assessment process. The interview included questions 
relating to the pregnancy (typical, rest, high-risk, stressful-traumatic), fertility treatment, delivery (vaginal, caesarean and instrumental) and prematurity to detect prenatal and perinatal problems.

\section{Participants}

This study is a preliminary research and have involved a convenience sample of 69 children between the ages of 3 and 11 obtained from a private occupational therapy centre in A Coruña (Spain). The sample was mainly male $(\mathrm{n}=55,79.71 \%)$ and met the following inclusion criteria: aged between 3 and 11 years, fully responding from SP-2 and having sensory processing difficulties detected by an ASI-trained occupational therapist. Of the total sample, $45(65,21 \%)$ children have had any prenatal or perinatal problem. Of the total sample, 10 children had an ASD diagnosis. The characteristics of the sample are shown in Table 1.

This sample was estimated to measure the impact of prematurity on children with sensory reactivity and children with sensory reactivity and co-occurring ASD, assuming a significance level of $\alpha=0.05$ (95\% confidence interval) and a maximum margin of sampling error of $<10 \%$ [5].

\section{Procedures}

To analyses the possible differences between the sensory factors and quadrants of the SP-2 [15] and the study variables: pregnancy (typical, high-risk, stress-traumatic), fertility treatment, delivery (vaginal, caesarean and instrumental) and prematurity, the Student's $t$-test or the Wilcoxon test for independent samples was used, depending on whether or not the hypothesis of normality was verified (Shapiro-Wilk test). If the groups to be compared had three or more variables, the ANOVA test or the Kruskal-Wallis test was used, depending on the hypothesis of normality and homocedasticity. The significance level used was 0.05 . The statistical analysis was carried out using R (R Development Core Team), version 3.6.0.

\section{Results and Discussion}

This study has obtained statistically significant differences in the analysis of visual processing and body position, as well as in the emotional problems derived from a sensory processing dysfunction in children with perinatal and postnatal sensory processing problems.

In relation to visual processing, statistically significant differences have been obtained depending on the type of pregnancy. The ANOVA test has obtained a p-value of 0.007 ; specifically, Tukey's post-hoc test detects differences when comparing stressful and traumatic pregnancy with natural pregnancy ( $\mathrm{p}$-value $=0.003$ ).

In relation to body-position processing, statistically significant differences have been obtained depending on prematurity. The Student's $t$-test obtained a p-value of $<0.001$ on comparing the bodyposition between premature children and children carried to term.

In relation to emotional problems, statistically significant differences have been obtained depending on the type of pregnancy. The ANOVA test obtained a p-value of 0.042 ; specifically, Tukey's post-hoc test detected differences when comparing natural pregnancy with high-risk pregnancy ( $\mathrm{p}$-value=0.026).

This study shows no statistically significant differences in auditory, tactile, movement (vestibular) or oral sensory processing,
Table 1: Sample characteristics.

\begin{tabular}{|c|c|c|}
\hline \multicolumn{2}{|c|}{ Variable } & \multirow{2}{*}{$\begin{array}{c}\text { n (\%) } \\
55(79.71)\end{array}$} \\
\hline & Male & \\
\hline 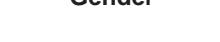 & Female & $14(20.29)$ \\
\hline \multirow{4}{*}{ Pregnancy } & Normal & $51(73.91)$ \\
\hline & Rest & 11 (15.95) \\
\hline & High-risk & $4(5.79)$ \\
\hline & Stressful/traumatic & $3(4.34)$ \\
\hline \multirow{2}{*}{ Fertility treatment } & Yes & $9(13.05)$ \\
\hline & No & $60(86.95)$ \\
\hline \multirow{3}{*}{ Delivery } & Vaginal & $38(55.07)$ \\
\hline & Caesarean section & $18(26.08)$ \\
\hline & Instrumental & 12 (17.39) \\
\hline \multirow{2}{*}{ Prematurity } & Yes & $7(10.14)$ \\
\hline & No & $61(88.40)$ \\
\hline
\end{tabular}

Table 2: Results for the prematurity variable.

\begin{tabular}{|l|c|c|c|}
\hline \multirow{2}{*}{\multicolumn{1}{|c}{ Sensory factor }} & \multicolumn{2}{|c|}{ Prematurity } & \multirow{2}{*}{ p-value } \\
\cline { 2 - 4 } & No & Yes & \\
\hline Hearing processing & $20.02(7.42)$ & $21.14(6.99)$ & 0.704 \\
\hline Visual processing & $13.32(5.49)$ & $14.86(5.37)$ & 0.486 \\
\hline Tactile processing & $20.97(8.11)$ & $21.86(7.47)$ & 0.783 \\
\hline Movement processing & $16.39(7.28)$ & $19.00(5.20)$ & 0.362 \\
\hline Body position & $11(8-17.50)$ & $8(8-8,5)$ & 0.026 \\
\hline Oral processing & $20.15(8.99)$ & $23.14(12.33)$ & 0.427 \\
\hline Behaviour & $20.32(9.02)$ & $22.43(2.88)$ & 0.2 \\
\hline Socio-emotional & $34.34(13.28)$ & $36.86(16.26)$ & 0.781 \\
\hline Attention & $26.64(9.42)$ & $26.00(9.57)$ & 0.865 \\
\hline Low registration & $43.59(15.41)$ & $40.14(12.21)$ & 0.57 \\
\hline Sensory seeking & $41.93(16.13)$ & $45.43(5.22)$ & 0.237 \\
\hline Sensory sensitivity & $42.71(12.87)$ & $45.29(10.80)$ & 0.614 \\
\hline Sensory avoiding & $47.88(15.28)$ & $50.29(13.20)$ & 0.692 \\
\hline Note p-vale refer & & & \\
\hline
\end{tabular}

Note: $\mathrm{p}$-value referred to Student or Welch $t$-test, or Wilcoxon test.

or in attention and behaviour problems derived from a sensory processing dysfunction in the variables studied. No statistically significant differences were detected when analyzing the sensory quadrants established in Dunn's Model. Tables 2 and 3 shows the results obtained in this study.

This study aims to identify differences in sensory processing in children with sensory reactivity difficulties (both with and without prenatal and perinatal problems).

The results obtained in this research indicate that there are statistically significant differences in sensory processing in children who have suffered problems in pregnancy or who have been premature. Specifically, statistically significant differences have been identified in visual sensory processing, in body position and in emotional difficulties in children who have undergone a stressful pregnancy, premature children and children who have had high-risk pregnancy, respectively. 
Table 3: Results according to pregnancy. Different letters indicate significant differences.

\begin{tabular}{|c|c|c|c|c|c|}
\hline \multirow{2}{*}{ Sensory factor } & \multicolumn{4}{|c|}{ Pregnancy } & \multirow{2}{*}{ p-value } \\
\hline & Typical & Rest & High-risk & Stressful-traumatic & \\
\hline Hearing processing & $19.72(7.81)$ & $20.00(7.36)$ & $14.25(9.74)$ & $27.33(3.06)$ & 0.19 \\
\hline Visual processing & $13.33(5.28)^{a, b}$ & $13.36(5.28)^{\mathrm{a}}$ & $6.25(4.27)^{a, b}$ & $20.67(6.66)^{\mathrm{b}}$ & 0.007 \\
\hline Tactile processing & $20.89(8.20)$ & $19.18(7.22)$ & $17.50(14.15)$ & $29.00(5.57)$ & 0.282 \\
\hline Movement processing & $16.67(7.71)$ & $15.45(5.91)$ & $10.50(7.19)$ & 23.67 (3.79) & 0.133 \\
\hline Body position & $11.5(8-17.15)$ & $12(8-17.5)$ & $8(6-8.50)$ & $8(8-17.75)$ & 0.129 \\
\hline Oral processing & $19.39(8.77)$ & $21.18(9.15)$ & $16.00(17.05)$ & $24.67(12.86)$ & 0.636 \\
\hline Behaviour & $20.50(9.64)$ & $20.55(6.71)$ & $13.50(9.26)$ & $24.33(5.51)$ & 0.42 \\
\hline Socio-emotional & $36.46(14.25)^{b}$ & $36.45(8.66)^{a, b}$ & $15.75(18.95)^{b}$ & $31.67(12.66)^{a, b}$ & 0,042 \\
\hline Attention & $26.57(9.72)$ & $27.00(10.89)$ & $19.75(14.17)$ & 24.33 (8.39) & 0.609 \\
\hline Low registration & 44.07 (16.22) & $42.64(14.75)$ & $25.00(18.06)$ & $44.00(6.56)$ & 0.158 \\
\hline Sensory seeking & 41.65 (16.61) & 40.09 (13.09) & $32.30(25.01)$ & $52.33(10.12)$ & 0.467 \\
\hline Sensory sensitivity & $42.83(12.82)$ & $41.27(15.06)$ & $28.50(19.67)$ & 51.33 (8.08) & 0.143 \\
\hline Sensory avoiding & $48.26(16.04)$ & $50.00(10.13)$ & $27.50(26.66)$ & 51.67 (9.71) & 0.082 \\
\hline
\end{tabular}

Note: $\mathrm{p}$-value of ANOVA or Kruskal-Wallis test.

Studying sensory reactivity within the field of Occupational Therapy (OT) is essential, as problems with self-regulation have been associated with difficulties in social skills and academic competence and behavioural problems $[17,18]$. Authors have even linked sensory reactivity problems to mental health problems in adulthood [19].

Various research studies have found that prenatal distress and stress can interfere with self-regulation [20] and negatively affect a baby's neurological development [18,21]. Following this vein, the present study has found that children who have experienced prenatal distress show statistically significant differences in visual processing versus children with sensory processing difficulties who have not. The findings of Jafari et al. [12] indicate that prenatal stress produces a fourfold increase in basal corticosterone and reduced amplitude of all cortical sensory responses evoked, including visual and auditory responses.

Other results obtained in this study were statistically significant differences between the pregnancy variable (specifically, high-risk pregnancy) and emotional problems derived from inadequate sensory processing. These findings support the results of O'Connor, Heron, Golding, Glover \& AL Spac Study Team [22] who has obtained that regulatory problems have been linked to problems in social-emotional development and behavioural problems. With this, it can be expected that the sensory regulation problems obtained using the SP-2 sensory questionnaire [15] will yield statistically significant scores in factors such as the emotional state of children with sensory processing problems. Bush et al. [23] have showed than mothers who felt more stressed during pregnancy and postpartum indicated that their babies had a difficult temperament and less ability to self-regulate. Stress during pregnancy, especially in early pregnancy, results in delayed fetal maturation, altered emotional regulation and impaired cognitive performance during childhood [21].

Probably due to the small sample used in this research, this study has not yielded statistically significant differences between children who have suffered from a stressful/traumatic pregnancy and tactile and auditory sensory processing, expected results following the findings obtained by Keuler et al. [24], who drew a relationship between prenatal complications and problems of auditory and tactile hyperreactivity. These results [30] are a finding compatible with the research of Schneider et al. [11] in rhesus monkeys, thus suggesting that prenatal maternal stress increases babies' tactile sensitivity, and the study by Heuvel, Donkers, Winkler, Otte \& Van Der Bergh [25], which indicates that greater maternal anxiety could be interpreted as a reflection of weaker habituation to auditory stimuli in these babies, thus indicating less adaptive brain functioning.

This study has found that children born prematurely have greater problems in proprioceptive processing (body position) than children carried to term who have problems in sensory processing. Previous research studies have linked prenatal depression to an increased likelihood of preterm birth and, in turn, preterm birth to increased sensory processing deficiencies [26] and sensory-motor development [27]. Babies of mothers with prenatal depression or anxiety suffer from more hypotonia in the neonatal period [28]. Ryckman, Hilton, Rogers and Pineda [29] conclude that premature infants are at an increased risk of developing a sensory processing disorder, with results indicating that $50 \%$ of premature infants have sensory processing problems.

With the aim of determining whether preterm infants have atypical responses to sensory stimuli, Wickremasinghe, Rogers, Johnson, Shen, Barkovich and Marco [30] conducted a study similar to this study by administering the Sensory Profile [31] sensory questionnaire to 107 infants born at 32 weeks. The results of Wickremasinghe et al. [30] indicated that $39 \%$ of the children evaluated had at least one sensory factor or quadrant with a deviation of $-2 \mathrm{SD}$, particularly auditory, tactile and vestibular factors. Along the same lines, Cabral, Pereira da Silva, Martinez \& Tudella [26] show that premature infants present with differences in tactile and vestibular processing and Bart, Shayevits, Gabis \& Morag [32], using the Infant/Toddler Sensory Profile [33], found that premature babies are more likely to have sensory reactivity problems, specifically in auditory and oral processing. 
The results of the present study do not yield significant correlation between the other sensory factors analyzed using the SP-2 [15] and the prematurity variable. This may be due to the fact that the sample size is small and the group of children born prematurely vs. children born at term is not balanced and, therefore, there may be difficulties in detecting significant differences, despite the fact that the effect size is medium and large in several sensory factors.

However, the results obtained in this study indicate that the prevalence of prematurity in children with sensory reactivity problems with and without ASD was $10.2 \%$. This result is similar to the prevalence found by May-Bensson et al. [5], who found that between 12.4-16.0 \% of children with DIS with and without an ASD diagnosis were children born prematurely.

Regarding the birth variable, it should be noted that the results of this study indicate similar data to those found by May-Bensson et al. [5]. This study shows that $43 \%$ of the children had an instrumental delivery, which is closely in line with May-Bensson et al. [5] who found that $36.1 \%$ of the children with sensory reactivity and $43.5 \%$ of the children with sensory reactivity and ASD needed some kind of assistance during delivery.

The results of this and other similar studies are relevant since previous research studies indicate that children with sensory reactivity problems at younger ages score significantly higher at older ages and are more likely to have a clinical diagnosis $[34,35]$. Examples can be found in the study by DeGangi, Breinbauer, Doussard-Roosevelt, Porges and Greenspan [34] which, when analyzing children between 7 and 30 months and then at 36 months of age, still observed sensory reactivity problems, and the results of Schneider et al. [35] in rhesus monkeys, which found that neonatal sensory processing function correlates with adult tactile sensory function, indicating continuity of sensory processing function over a wide age range.

The relationship between prenatal and perinatal problems and sensory processing difficulties persisting at older ages demonstrates the need for early identification and treatment of such problems in public health services. Early identification of sensory processing problems in these children could anticipate interventions from disciplines such as occupational therapy in neonatal and early care units at public and private hospitals, thus improving children's self-regulation, sensory-motor development and the acquisition of functional skills.

One of the limitations of this study is the small convenience sample used, accordingly, the results should be interpreted with caution. This study has assumed a margin of error greater than $5 \%$ since it is a pilot study that aims to initiate a line of research, and given the difficulty of access to the sample since, although the prevalence of sensory reactivity problems in Spain in typically developing children is between $9.5 \%$ and $13 \%$, it is still an under-diagnosed condition [36].

However, this research sets the stage for future work that can explore these hypotheses in a larger sample.

Another limitation has been the lack of adapted, validated and standardized measures in Spain to analyze and detect prenatal distress problems. The use of standardized assessment tools in future studies could yield promising new results.

\section{Conclusion}

These results confirm that there are differences in the sensory processing of children with sensory processing disorders who have suffered perinatal and postnatal problems compared to those who have not. Prenatal and perinatal factors like a prematurity or pregnancy are predictive factors for sensory processing disorders.

\section{Acknowledgment}

Thanks to the families that have participated. Thanks to the Scientific and Technical Services consultancy unit of the Universidad de Oviedo, and particularly to Tania Iglesias, for methodological advice. Research carried out by the Unidad de Integración y Promoción de la Salud (Integra-Saúde), Universidade da Coruña.

\section{References}

1. Ayres AJ. The development of perceptual-motor abilities: A theoretical basis for treatment of dysfunction (Eleanor Clarke Slagle Lecture). Am J Occup Ther. 1963; 17: 221-225.

2. May-Benson TA, Doomar JA, Teasdale A. Incidence of pre-, peri-, and postnatal birth and developmental problems of children with sensory processing disorder and children with autism spectrum disorder. Front Integr Neurosci. 2009; $3: 31$.

3. Dunn W, Westman K. The sensory profile: the performance of a national sample of children without disabilities. Am J Occup Ther. 1997; 51: 25-34.

4. Ahn RR, Miller LJ, Milberger S, McIntosh DN. Prevalence of parents' perceptions of sensory processing disorders among kindergarten children. Am J Occup Ther. 2004; 58: 287-293.

5. Delgado-Lobete L, Montes-Montes R, Seoane SR. Prevalencia de Trastorno del Procesamiento Sensorial en niños españoles. Resultados preliminares y comparación entre herramientas de diagnóstico. Revista electrónica de terapia ocupacional Galicia, TOG. 2016; 24: 5.

6. Ben-Sasson A, Hen L, Fluss R, Cermak SA, Engel-Yeger B, Gal E. A metaanalysis of sensory modulation symptoms in individuals with autism spectrum disorders. J Autism Dev Disor. 2009; 39: 1-11.

7. Lane AE, Young RL, Baker AEZ, Angley MT. Sensory processing subtypes in autism: Association with adaptive behavior. J Autism Dev Disor. 2010; 40: 112-122.

8. Schneider ML, Moore CF, Gajewski LL, Larson JA, Roberts AD, Converse AK, et al. Sensory processing disorder in a primate model: Evidence from a longitudinal study of prenatal alcohol and prenatal stress effects. Child Dev. 2008; 79: 100-113.

9. Jafari Z, Rezaei Z, Afrashteh N, Torabi R, Singh S, Kolb BE, et al. Prenatal Stress Dysregulates Resting-State Functional Connectivity and Sensory Motifs. BioRxiv. 2020.

10. Case-Smith J, Butcher L, Reed D. Parents' report of sensory responsiveness and temperament in preterm infants. Am J Occup Ther. 1998; 52: 547-555.

11. Dunn W. Sensory Profile 2. User's Manual. Strengths-Based Approach to Assesment and Planning. San Antonio: The Psychological Corporation. 2014.

12. Dunn W. The impact of sensory processing abilities on the daily lives of young children and their families: A conceptual model. Infants Young Child. 1997; 9: 23-35.

13. Weinstock M. Does prenatal stress impair coping and regulation of hypothalamic-pituitary-adrenal axis?. Neurosci Biobehav Rev. 1997; 21: 1-10.

14. Gándara-Gafo B. Adaptación cultural, validación y valores de referencia del Adolescent/Adult Sensory Profile en España. Universidade da Coruña (Spain). 2016.

15. Lin B, Crnic KA, Luecken LJ, Gonzales NA. Maternal prenatal stress and infant regulatory capacity in Mexican Americans. Infant Behav Dev. 2014; 37: 571-582. 
16. Sandman CA, Davis EP, Buss C, Glynn LM. Exposure to prenatal psychobiological stress exerts programming influences on the mother and her fetus. Neuroendocrinol. 2012; 95: 8-21.

17. O'Connor TG, Heron J, Golding J, Glover V, AL Spac Study Team. Maternal antenatal anxiety and behavioural/emotional problems in children: a test of a programming hypothesis. J Child Psychol Psychiatry. 2003; 44: 1025-1036.

18. Bush NR, Jones-Mason K, Coccia M, Caron Z, Alkon A, Thomas M, et al. Effects of pre-and postnatal maternal stress on infant temperament and autonomic nervous system reactivity and regulation in a diverse, low-income population. Development and psychopathology. 2017; 29: 1553-1571.

19. Keuler MM, Schmidt NL, Van Hulle CA, Lemery-Chalfant K, Goldsmith $\mathrm{HH}$. Sensory over-responsivity: prenatal risk factors and temperamental contributions. J Dev Behav Pediatr. 2011; 32: 533.

20. Van den Heuvel M I, Donkers FC, Winkler I, Otte RA, Van den Bergh BR. Maternal mindfulness and anxiety during pregnancy affect infants' neural responses to sounds. Soc Cogn Affect Neurosci. 2015; 10: 453-460.

21. Cabral TI, da Silva LGP, Martinez CMS, Tudella E. Analysis of sensory processing in preterm infants. Early Hum Dev. 2016; 103: 77-81.

22. Charkaluk ML, Truffert P, Fily A, Ancel PY, Pierrat V, Epipage Study Group. Neurodevelopment of children born very preterm and free of severe disabilities: the Nord-Pas de Calais Epipage cohort study. Acta Paediatr. 2010; 99: 684-689.

23. Conradt E, Lester BM, Appleton AA, Armstrong DA, Marsit CJ. The roles of DNA methylation of NR3C1 and $11 \beta-H S D 2$ and exposure to maternal mood disorder in utero on newborn neurobehavior. Epigenetics. 2013; 8: 1321 1329.
24. Ryckman J, Hilton C, Rogers C, Pineda R. Sensory processing disorder in preterm infants during early childhood and relationships to early neurobehavior. Early Hum Dev. 2017; 113: 18-22.

25. Wickremasinghe AC, Rogers EE, Johnson BC, Shen A, Barkovich AJ, Marco EJ. Children born prematurely have atypical sensory profiles. Am J Perinatol. 2013; 33: 631-635.

26. Dunn W. Sensory Profile. San Antonio, TX: The Psychological Corporation. 1999.

27. Bart O, Shayevits S, Gabis LV, Morag I. Prediction of participation and sensory modulation of late preterm infants at 12 months: A prospective study. Res Dev Disabil. 2011: 32: 2732-2738.

28. Dunn W. Infant/Toddler Sensory Profile. San Antonio, TX: The Psychological Corporation. 2002

29. DeGangi GA, Breinbauer C, Roosevelt JD, Porges S, Greenspan S. Prediction of childhood problems at three years in children experiencing disorders of regulation during infancy. Infant Mental Health Journal: Official Publication of the World Association for Infant Mental Health. 2000; 21: 156-175.

30. Schneider ML, Moore CF, Adkins M, Barr CS, Larson JA, Resch LM, Roberts A. Sensory processing in rhesus monkeys: developmental continuity, prenatal treatment, and genetic influences. Child Dev. 2017; 88: 183-197.

31. Delgado-Lobete L, Pértega-Díaz S, Santos-del-Riego S, Montes-Montes R. Sensory processing patterns in developmental coordination disorder, attention deficit hyperactivity disorder and typical development. Res Dev Disabil. 2020; 100: 103608. 